\title{
Simultaneous conjugate ground-satellite ULF pulsation measurements at the middle latitudes
}

\author{
K. Prikner \\ Geophysical Institute, Academy of Science of the Czech Republic Prague, Boční II, 14131 Praha 4-Spořilov, Czech Republic
}

Received: 17 July 1996 / Revised: 14 January 1997 / Accepted: 5 February 1997

\begin{abstract}
The spectra of three series of simultaneous ground (Budkov Observatory) and satellite (Freja) ULF measurements have been processed. The following features were studied in the FFT spectra, power-spectra and cross-spectra of the six-minute samples, recorded during quasi-conjugate approaches of Freja to the local Budkov magnetic field line: (a) the Doppler frequency shift (about $1 \mathrm{mHz}$ and less) in the predominant and some particular spectral components on Freja; (b) the amplitude relations between the ground and satellite (ratios 0.3 to 0.8 ); (c) phase shifts between particular signal components on the spacecraft above the ionosphere (in the azimuthal $y$-direction on Freja) and on the ground (in the meridional $x$-direction at Budkov) have been estimated (phase lags on the ground $\leq 0.9 \mathrm{rad}$ ) and (d) also dynamical characteristics of the fundamental wave modes in the $(x, y)$-plane, perpendicular to the magnetic field line, have been estimated for the predominant spectral components, considering standing oscillation along the field line. Azimuthal wave numbers were about 2.0, propagating northwest at $\sim 400 \mathrm{~km} / \mathrm{s}$.
\end{abstract}

\section{Introduction}

ULF geomagnetic pulsations in the Pc3-5 frequency range $(f<50 \mathrm{mHz})$ are generally accepted as standing multiharmonic oscillations of the geomagnetic field lines (a review in Yumoto, 1986; Anderson, 1993; Cummings et al., 1969; Southwood, 1974; Takahashi and McPherron, 1984). This mechanism is not limited to the dayside hemisphere, where the Pc3 pulsations are likely generated as higher harmonics. The global spacial structure of the resulting harmonic oscillation arises from standing oscillations along the magnetic field line, especially the shear (toroidal) Alfvén wave, propagating in the $(x, y)$ plane perpendicular to the field lines (Walker et al., 1982). According to Samson et al. (1995) the time structure of the spectral bands of individual harmonics is likely due to field line resonances driven by wave guide modes with turning points within the plasmasphere and reflection at the dayside magnetopause.

Models of global resonances display coupling between compressional and toroidal modes indicating that the set of resonances is readily excited by broadband compressional power (Allan et al., 1987). In addition, field line resonances whose frequencies match global mode frequencies, are predicted to be strongly enhanced due to the retention of compressional energy in the global modes which damp via coupling to field line resonances (Allan and Poulter, 1989).

Hitherto ULF waves have been studied using measurements on the ground and on geostationary spacecraft (ATS 1,6; GOES 2-6, SMS 1), and also on elliptical orbits of HEOS-1, OGO-3,5, ISEE, AMPTE/ CCE (Anderson, 1993; Engebretson et al., 1986; Singer et al., 1982; Yumoto and Saito, 1983). In this article we devote ourselves to simultaneous measurements at $\sim 1700 \mathrm{~km}$ altitude on the Freja satellite (Freja magnetic field experiment team, 1994) made along sections of its orbit above the upper boundary of the ionosphere, and on the ground at the quasi-conjugate point with parts of these sections (at the Budkov Observatory).

The optimal sample time for ULF signal measurements by Freja appeared to be not longer than $6 \mathrm{~min}$ (shorter time makes the value of the FFT processing worse in the ULF range). With longer times (due to the satellite motion in both geographical longitude and latitude) distortions of the signal spectra will increase. The analyses show that during near-conjugate approaches of the satellite to Budkov these distortions are not substantial and the signal on the satellite and on the ground retains its basic frequency-amplitude structure. But in view of the relatively low velocities of both the shear and compressional wave modes of the standing oscillation structure, the Doppler effect in the Freja signal spectra may be important. Actually, frequency shifts relative to the (stationary) frequency on the ground have been found in the Freja spectra, and we 
have identified these with the Doppler effect (also see the comment in Sect. 5).

The simultaneous measurements of the signal above the ionosphere and on the ground allow ionospheric filtration effects to be specified. The wave directly measured both on the satellite and on the ground is always the total wave comprising both the coupled $R$ - and $L$-polarized modes propagating down from the upper layers of the ionosphere and also both modes reflected from the lower layers and the ground. The numerical method of ionospheric filtration modelling has been discussed by Prikner and Vagner (1983, 1985a, b, 1986).

In the Pc3-5 frequency range the ionosphere appears to be a very thin layer relative to the signal wavelength. Electric Hall currents play a major part in signal transmission to the ground. According to Hughes and Southwood (1976a), Glassmeier (1988) and Wedeken et al. (1984), a reduction factor, $g=\left(\sum_{H} / 2 \sum_{P}\right) \exp (-x$ $\left.\left|k_{\perp}\right| h\right)$, is used to describe the signal amplitude transmitted to the ground through the ionosphere. Here $\sum_{H}$ and $\sum_{P}$ are the height-integrated Hall and Pedersen conductivities, $x$ is the reduction factor of the scale lengths mapped from the equatorial plane down to the ionosphere, and $h$ is the height of the damping ionosphere. The quantity $k_{\perp}$ is the azimuthal component of the signal wave vector. In the Pc3-4 range analyzed, according to Glassmeier (1988) and Wedeken et al. (1984) the values of $g$ can range from 0.2 to 0.9 , and these results agree with the simulations of Prikner (1996), applying the numerical method of Prikner and Vagner (1983) and Prikner (1984).

The counterclockwise rotation through $90^{\circ}$ of the magnetic field polarization ellipse for the Alfvén-type (shear) oscillation propagating down through the ionosphere to the ground (Nishida, 1964; Hughes, 1974) is well known. This effect has also been numerically modelled by Prikner and Vagner (1985b) in the Pc1 frequency range. The ionospheric rotation angle can significantly deviate from $90^{\circ}$ due to the non-uniform horizontal conductivity distribution and, in terms of propagation of hydromagnetic waves, in non-coplanar magnetic field geometries (e.g., Glassmeier and Junginger, 1987; Glassmeier, 1988).

The purpose of this study is to outline some phenomena of the ionospheric transmissivity in the ULF range on the basis of simultaneous and quasiconjugate pulsation measurements at the Freja satellite and single ground observatory. By applying FFT spectral, power-spectral, and cross-spectral analysis to three selected pulsation events we have attempted to estimate the amplitudes of particular spectral pulsation components at both sites (i.e., ionospheric transmissivity), the Doppler frequency shift due to the satellite motion, and the phase relation between the characteristic (Alfvén) signal in the azimuthal $y$-component on the satellite and the signal in the north-south $x$-component on the ground (i.e., signal phase shift).

In addition, an attempt to illustrate propagation of the standing wave structure in the plane perpendicular to the magnetic field lines will be made for the principal components which occur in the spectra of the pulsation samples analyzed.

\section{Experimental data and processing}

The synchronous ULF pulsation measurements at the Budkov Observatory (geomagnetic latitude and longitude are $\Phi=49^{\circ} 01^{\prime}, \Lambda=96^{\circ} 02^{\prime}$; geographic latitude $\phi=49.08^{\circ} \mathrm{N}$ and longitude $\lambda=14.02^{\circ} \mathrm{E}$ ) and on the Freja satellite at the quasi-conjugate orbital sections (around $L_{F} \approx L_{B} \approx 2.3$ ) were made during May and September 1993. Freja was in a subpolar orbit with inclination $63^{\circ}$, and in the Northern Hemisphere at altitudes around $1700 \mathrm{~km}$ (apogee $1767 \mathrm{~km}$, perigee $\approx 600 \mathrm{~km}$ ). The orbital period was about $110 \mathrm{~min}$.

The relatively fast motion of the satellite in the lowaltitude orbit limited the effective time interval of quasiconjugate measurements in the ULF range to no more than $6 \mathrm{~min}$. During this interval the satellite changed its position in geographic latitude by about $5^{\circ}-10^{\circ}$ and in longitude by about $7^{\circ}-17^{\circ}$ (for details refer to Sect. 4).

The signal on Freja was recorded by magnetometers in three components of the mean field-aligned coordinate system, $z \| B_{0}, x$ pointing to the north and $y$ to the east. At the Budkov Observatory only the north-south $x$-component was recorded by a Bobrov-type magnetometer (system consists of a magnetic needle on stretched silicon fibres, photodiodes and an amplifier with digital equipment developed in the Geophysical Institute in Prague). However, according to Nishida (1964) and Hughes (1974), this component should correlate best with the Alfvén shear azimuthal component in the magnetosphere, considering the counterclockwise $90^{\circ}$ field rotation in the ionosphere.

\subsection{Signal processing for the FFT analysis}

To analyze frequency-phase and frequency-amplitude relations between the signal on Freja and on the ground, the spectra of the $x$ - and $y$-components of the Freja records were processed by the fast Fourier transformation technique (FFT). Undesirable long-period variations with large amplitudes (e.g., due to motion through the electrojet region) were eliminated by applying the least-squares filtration method using a polynomial of the 3rd degree.

The digital 6-min signal records with a time sampling $\delta t=1 \mathrm{~s}$ were FFT processed as in Prikner (1996). The resulting spectra with frequency sampling $\delta f \approx 1 \mathrm{mHz}$ (all the 1024 data points were used adding the zero values) can be expressed as

$S_{n}=M_{n} \exp \left(i \phi_{n}\right)$,

where $M_{n}$ are real spectral modules and $i \phi_{n}$ complex spectral phases in discrete frequencies $f_{n}$. The spectra were smoothed by tapering the signal edges with a cosine-bell window.

\subsection{Power-spectra and cross-spectra}

The FFT powerspectra of the Freja records, and on the ground, are calculated as $P F_{n}=F_{n} \cdot F_{n}^{*}$ and 
$P G_{n}=G_{n} \cdot G_{n}^{*}$, respectively, where $F_{n}$ and $G_{n}$ are FFT spectra (1) of the Freja and ground records, respectively, “*” indicates a complex conjugate value. The crossspectrum between the Freja record component and the ground is then $C_{n}=F_{n} \cdot G_{n}^{*}$. Using a rectangular smoothing window with a width of $2 \delta f \approx 2 \mathrm{mHz}$, the coherence can be defined as

Coherence $_{n}=\left|C_{n}\right| / \sqrt{\left(P F_{n} \cdot P G_{n}\right)}$.

It is also useful to express the complex values of the cross-spectrum as

$C_{n}=C M_{n} \exp \left(i \Psi_{n}\right)$,

where $C M_{n}$ are the values of the real cross-spectral modules and $i \Psi_{n}$ are the complex phases of the crossspectra. In agreement with the definition of the crossspectrum, the cross-spectral phase expresses the phase shift of the signal on Freja (index $F$ ) with respect to the signal on the ground $(G)$, i.e., $\Psi_{n}=\phi_{n}^{F}-\phi_{n}^{G}$, using Eqs. (1) and (3). $\Psi_{n}>0$ indicates that the signal on the ground lags in phase behind the signal on Freja.

\subsection{Estimation of amplitude and weighted frequency in a packet}

Real frequency components of the pulsation can be identified in the spectra of the conjugate signals. Their spectra are limited in frequency, $\Delta f=\left\langle f_{\min }, f_{\max }\right\rangle$. In analogy to Prikner (1996) it is useful to define the mean amplitude of the spectral packet in the considered recording component, using Eq. (1),

$A=\sum_{\Delta f} M_{n} \delta f \quad[n T]$.

The weighted frequency characterizes the corresponding spectral component of the pulsation,

$f_{W}=\sum_{\Delta f} f_{n} M_{n} / \sum_{\Delta f} M_{n}$

In selected conjugate events the limits of the $\Delta f$ intervals in both components $(x, y)$ on Freja and on the ground are very close and can be determined from the cross-spectral peaks.

\subsection{Estimation of the Doppler frequency shift}

The velocity of the Freja spacecraft at an altitude $\approx 1700 \mathrm{~km}$ during the measurements was $|u| \approx 7 \mathrm{~km} / \mathrm{s}$, in the $(x, y)$-plane with components $u_{x} \approx-4.5 \mathrm{~km} / \mathrm{s}$, $u_{y} \approx 5.4 \mathrm{~km} / \mathrm{s}$. The Doppler effect is significant, if the wave velocity components do not differ very much from the velocity components of the spacecraft (Baumjohann et al., 1987). Let us assume that the weighted frequency Eq. (5) on the ground is the stationary frequency of the pulsation component $\left(f_{G}\right)$. Then the weighted frequencies $f_{x}$ and $f_{y}$ characterize the coordinate components of the Doppler frequency shift on Freja, according to relations
$D f_{x}=f_{x}-f_{G}$

$D f_{y}=f_{y}-f_{G}$.

The corresponding Doppler changes of the wavelength, i.e. for $D f>0$ shortening and for $D f<0$ lengthening, is expressed by the formula

$\lambda / \lambda_{0}=f_{G} /\left(f_{G}+D f_{(x, y)}\right)=f_{G} / f_{(x, y)}$.

\subsection{Estimation of the phase shift between the signal on Freja and on the ground}

The cross-spectral phases of the analyzed pulsation samples show a slower change around the weighted spectral peak frequencies. Let us take the average frequency $\bar{f}$ of the weighted spectral peak frequencies $f_{x}, f_{y}$ and $f_{G}$ to be the characteristic frequency of the pulsation spectral component. The phase shift in $\bar{f}$ between the coordinate component on Freja and the ground can then be estimated.

Most of the analyzed spectral components in the Pc3-4 range show a prevailing azimuthal $y$-component in the signal record, providing evidence of the prevailing shear Alfvén-type oscillation. The signal phase shift between Freja and the ground can characterize at best the cross-spectral phase between the signal in the $y$-component on Freja and $x$-component on the ground. Then, $\Delta \phi_{y x}=\Psi_{y x}=\phi_{y}^{F}(\bar{f})-\phi_{x}^{G}(\bar{f})$, using the phases from Eqs. (1) and (3). The values $\Psi_{y x}>0$ are the phase lag of the signal on the ground behind that on Freja.

\section{Doppler frequency shift and wave characteristics}

At Freja, the oscillation of the $x$-component predominantly represents the compressional mode (c), and the $y$-component the shear Alfvén mode (s) of the $z$-standing wave oscillation structure. Both modes are generally coupled, have an identical cyclic frequency $\omega$, and we assume $\omega=\omega_{G}$ on the ground. The frequencies in both components on Freja are Doppler shifted: $\omega_{c}^{\prime}$ in the $x$-component and $\omega_{s}^{\prime}$ in the $y$-component. The velocity of the satellite at the point of measurement was $|\mu| \approx 7 \mathrm{~km} / \mathrm{s}, u_{x} \approx-4.5 \mathrm{~km} / \mathrm{s}, u_{y} \approx 5.4 \mathrm{~km} / \mathrm{s}$.

The Doppler shifted frequency $\omega^{\prime}$ and the real (stationary) frequency $\omega=\omega_{G}$ are related as in $\mathrm{Ba}-$ umjohann et al. (1987)

$\omega=\omega^{\prime}+\mu \cdot \kappa$,

where $\kappa$ is the wave vector of the particular mode. Then,

$\omega_{s}^{\prime}+\mu \cdot \kappa_{s}=\omega_{c}^{\prime}+\mu \cdot \kappa_{c}=\omega_{G}$.

In terms of the particular satellite coordinates, then

$\omega_{G}=\omega_{c}^{\prime}+u_{x} k_{c x}+u_{y} k_{c y}$

$\omega_{G}=\omega_{s}^{\prime}+u_{x} k_{s x}+u_{y} k_{s y}$. 
According to Walker et al. (1982), the $y$-component of the compressional mode coupled with the shear mode is controlled by the shear wave, so that

$k_{c y}=k_{s y}=k_{y}$.

According to Singer et al. (1982), for standing hydromagnetic waves under the condition that the shear wave is the fundamental mode, $k_{\|} \ll k_{x}, k_{y}$, and since $\nabla \cdot \beta=0$ ( $\beta$ is the instantaneous variation of the wave magnetic field), $k_{s x} b_{x}+k_{s y} b_{y}=0$. This equation in view of Eqs. (4), (5) and (11), determines the shear oscillation mode as

$k_{s x}=K \cdot k_{y}$,

where

$K= \pm A_{y} / A_{x}$

and $A_{x}, A_{y}$ (instead of $b_{x}$ and $b_{y}$ ) are the spectral amplitudes (Eq. 4), calculated for the $x$ - and $y$-Freja coordinates.

The sign of $K$ can be determined from the spectral phase shifts $\Delta \phi=\phi_{y}-\phi_{x}$ (Eq. 1) between both the coordinate components of this spectral component on Freja: "-" for $|\Delta \phi|<\pi / 2$ (oscillation in the 1st and 3rd quadrants), " + " for $\pi / 2<\Delta \phi<3 \pi / 2$ (oscillation in the 2nd and 4 th quadrants).

If relation (11) holds true, then Eqs. (10a, b) and (12) yield

$k_{y}=\left(\omega_{G}-\omega_{s}^{\prime}\right) /\left(K u_{x}+u_{y}\right)$,

$k_{c x}=\left(\omega_{G}-\omega_{c}^{\prime}-u_{y} k_{y}\right) / u_{x}$.

Equations (11)-(15) then determine the wave vectors for both particular wave modes, i.e., the wave characteristics of the standing oscillation structure propagating in the $(x, y)$-plane.

The polarization of the pulsation components $(R$ or $L)$ can also be determined from the $x$ - and $y$-spectral phases (Eq. 1). The $R$-polarization arises from the phase of the $x$-component signal leading that of the $y$-component, the opposite being true for the $L$-polarization.

The azimuthal wave number of the particular pulsation component at the position of the satellite is then defined as

$m=k_{y} L_{B} R_{E} \approx 14650 k_{y}$,

where $L_{B}=2.3\left(=L_{F}\right)$ is $L$ of the Budkov Observatory, and $R_{E}$ is the Earth's radius. It will be roughly estimated on the basis of coupled Freja-ground measurements of high-coherent signals, applying the Doppler effect in these equations.

The orientation of the $s$-wave-front relative to the positive direction of the $x$-axis is given by the wave-front slope angle,

$\alpha=-\arctan \left(k_{s x} / k_{y}\right)=-\arctan K$,

where $\alpha>0$ indicates a deflection of the wave-front to the east (oscillation in the 1 st and 3rd quadrants, $k_{s}$ pointing either to the 2 nd or 4 th quadrant depending on the sign of $k_{y}$ ).

\section{Case studies}

The ULF pulsation signal with frequency $f<50 \mathrm{mHz}$ was synchronously recorded on Freja and at Budkov during the spacecraft's approaches to the geomagnetically conjugate points during April-May 1993 and September 1993. Several events from the first interval were presented in Prikner (1996). Unfortunately, the simultaneous occurrence of clear, large pulsation events was very rare, mostly with poor conjugacy in longitude $\left(\Delta \lambda>10^{\circ}\right)$. In the second interval 6 events were analyzed, two of them, on 17 and 18 September, with favorable conjugacy (the last two minutes of the measurement in direct conjugacy). For comparison of results with poorer conjugacy the 13 May event will also be discussed here.

\subsection{September 1993, 02:56:00-03:02:00 UT}

Other details are $K_{p}=1_{-}, A_{p}=4$; Freja altitude $=1765$ to $1719 \mathrm{~km}$. The Freja field-line footprint was $\phi \approx 58.04^{\circ}$ to $47.94^{\circ}, \lambda \approx-0.09^{\circ}$ to $17.00^{\circ}$ (Budkov Observatory $\phi=49.08^{\circ}, \lambda=14.02^{\circ}$ ).

Tapered six-minute simultaneous magnetic field records in the $x$ - and $y$-components on Freja and in the $x$-component on the ground are shown in Fig. 1 . The

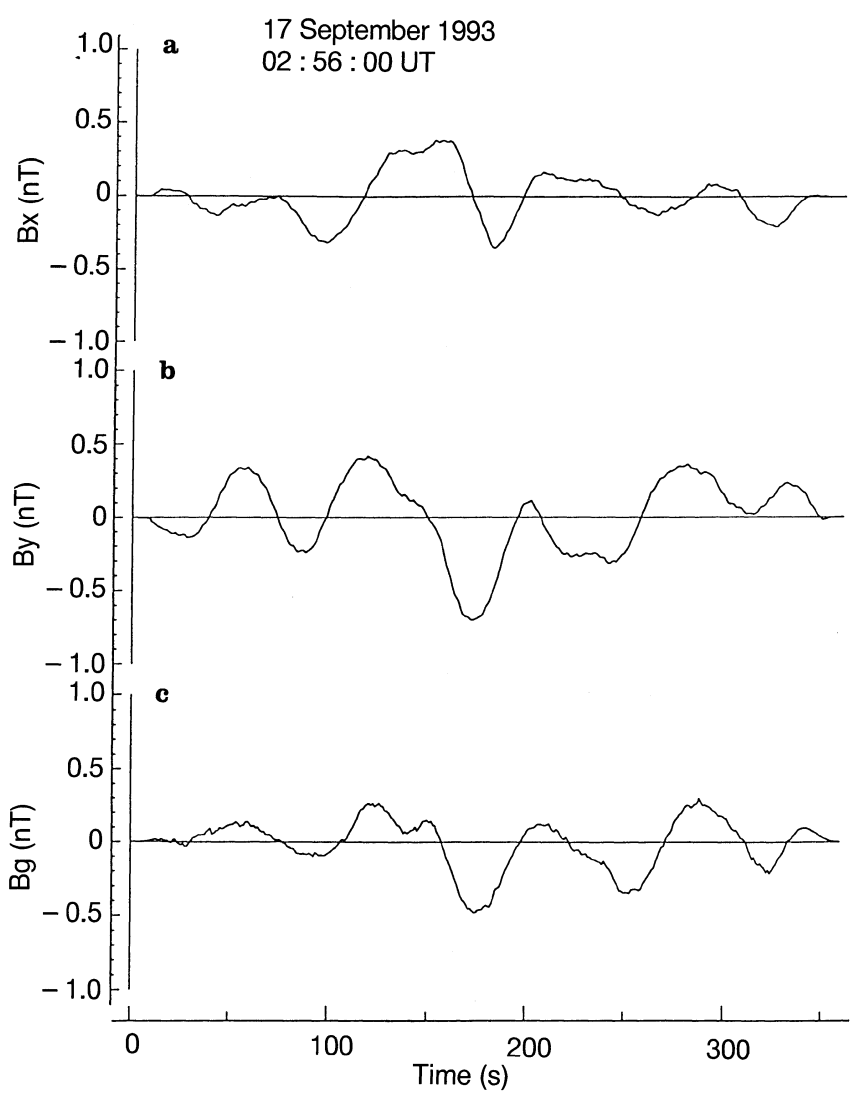

Fig. 1a-c. The signal B-field records on 17 September 1993, 02:56:00 03:02:00 UT, tapered by a cosine-bell window, a on Freja, $x$ component (N-S), b on Freja, $y$-component $(\mathrm{E}-\mathrm{W})$, c at Budkov, $x$ component 
vehicle spin period $T_{\text {spin }} \approx 6 \mathrm{~s}$ and nutation period $T_{\text {nut }} \approx 20 \mathrm{~s}$ were eliminated from the Freja records by signal smoothing with averaging sliding windows of $6 \mathrm{~s}-$ and 20 s-widths. Figure 1 shows a highly coherent signal at both points of measurement.

The FFT spectral analysis showed that the main predominant spectral component of the signal on Freja was in the $y$-component, and also that the total (compound) wave in the $(x, y)$-plane was $L$-polarized. Thus an Alfvén shear wave can be assumed. We can expect the phase characteristics of the oscillation in the $y$-component on Freja to correlate with the $x$-component on the ground, considering the $90^{\circ}$ counterclockwise rotation of the wave field in the ionosphere.

The associated display of the signal power-spectra in $y$-Freja and in $x$-Budkov for this event is given in Fig. 2a. The spectra of the real cross-spectral modulus and its phase (Eq. 3) for $y$-Freja and $x$-Budkov are

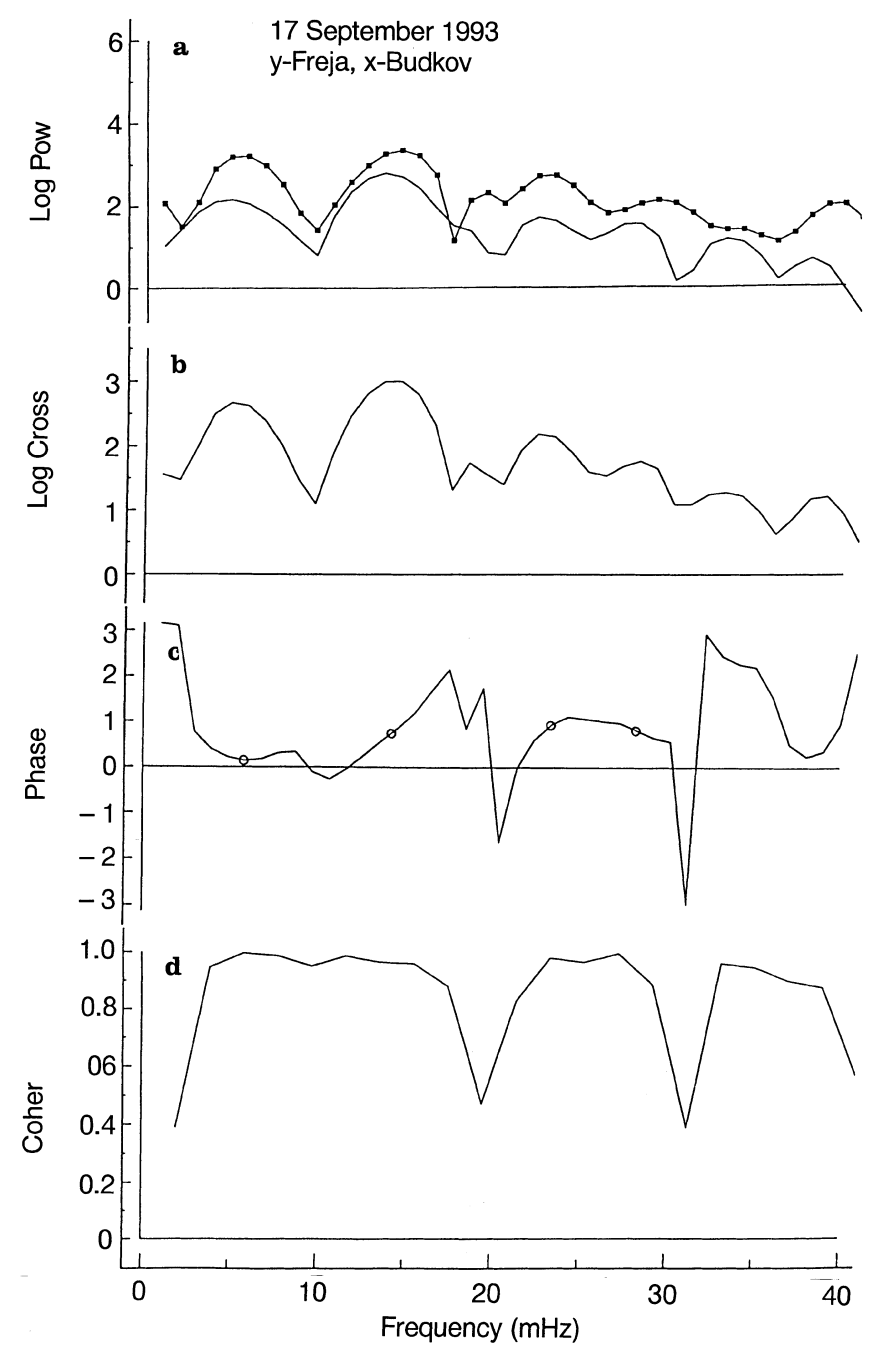

Fig. 2a-d. Frequency dependences for the event of 17 Sept. 1993: a associated power-spectra (on a logarithmic scale of $\mathrm{nT}^{2} / \mathrm{Hz}$ ) for $y$ Freja (line with squares) and $x$-Budkov (continuous line). b The real modulus of the $y$-Freja and $x$-Budkov cross-spectrum $\left(\log \mathrm{nT}^{2} / \mathrm{Hz}\right)$. c the phase of the $y$-Freja and $x$-Budkov cross-spectrum (on rad), and d the coherence calculated for the $y$-Freja and $x$-Budkov crossspectrum with a smoothing frequency window $2 \mathrm{mHz}$ in width shown in Fig. 2b, c. The coherence (Eq. 2) dependence on frequency is shown in Fig. 2d. Comparisons between the $x$-Freja and $x$-Budkov components by means of similar spectra are shown in Fig. 3a-d.

a. In this night-time event the predominant spectral component is at Pc4 frequencies. The weighted frequencies (Eq. 5) for the spectral peaks are $f_{G}=$ $13.83 \mathrm{mHz}\left(T_{G}=72.3 \mathrm{~s}\right)$ on the ground, $f_{y}=14.07$ $m H z\left(T_{y}=71.1 \mathrm{~s}\right), \quad f_{x}=13.30 \mathrm{mHz} \quad\left(T_{x}=75.2 \mathrm{~s}\right) \quad$ on Freja. Considering the Doppler effect on Freja, according to Eq. (6a, b), $D f_{y}=0.24 \mathrm{mHz}$ and $D f_{x}=$ $-0.53 \mathrm{mHz}$. In view of Eq. (7) the wavelength in $y$ Freja should be shortened by multiplying with the coefficient 0.98 , in $x$-Freja lengthened by multiplying with the coefficient 1.04 . This indicates a very slight Doppler distortion.

The amplitude relations for this particular predominant spectral component according to Eq. (4) are $A_{y}=0.22 \mathrm{nT}, A_{x}=0.16 \mathrm{nT}$ on Freja, $A_{G}=0.12 \mathrm{nT}$ on the ground. The ionospheric wave damping can then be characterized by the coefficient (transmission ratio),

$$
R_{G / F}=A_{G} \sqrt{2} / \sqrt{\left(A_{x}^{2}+A_{y}^{2}\right)}
$$

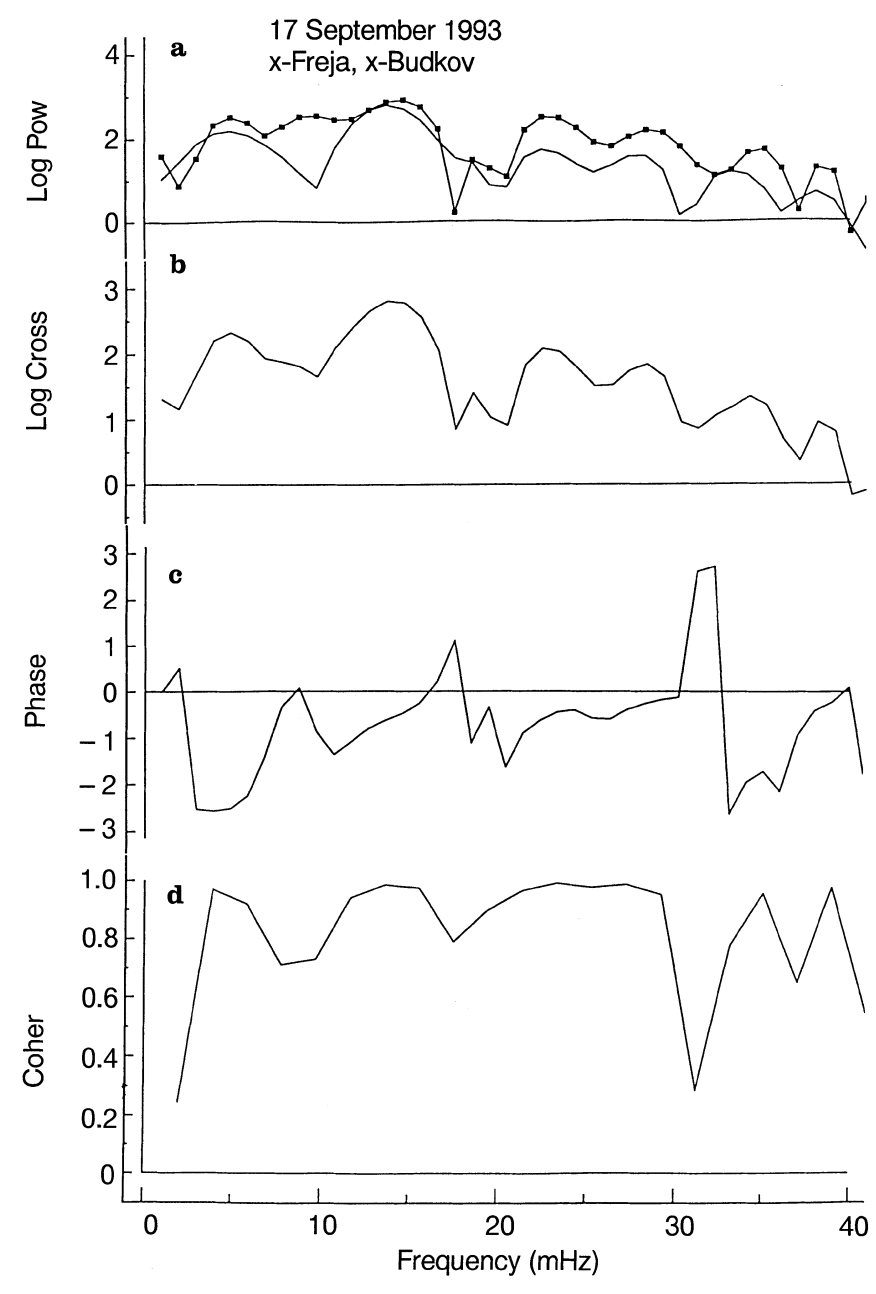

Fig. 3a-d. Frequency dependences analogous to Fig. 2, but for the $x$ Freja and $x$-Budkov signal records of 17 Sept. 1993 
where the ground amplitude in the $x$-component is considered to be modified by the expected complement of the $y$-amplitude in analogy with Prikner (1996). In this case $R_{G / F}=0.6$.

The cross-spectral phase (Fig. 2c) around the weighted peak frequencies characterizes the phase difference between the signal on the satellite and on the ground. For the mean frequency $\bar{f}=13.78 \mathrm{mHz}(\bar{T}=72.6 \mathrm{~s})$ of the predominant spectral component the cross-spectral phase (Eq. 3) between $y$-Freja and $x$-Budkov is $\Psi_{y x}(\bar{f})=0.61 \mathrm{rad}$ (marked in Fig. 2c). This indicates a phase lag of $0.61 \approx 0.2 \pi$ in the signal on the ground. These calculated values are arranged in Table 1 together with others for the next analyzed spectral components.

b. A similar analysis can be carried out for surrounding spectral components not distorted very much by signal filtration (as very low frequencies) or by their own low power. From the higher frequency side the cut-off frequency of the spectral processing appears to be the satellite spin nutation frequency $\left(f_{\text {nut }} \approx 50 \mathrm{mHz}\right)$. The low frequency component (between $\mathrm{Pc} 4-5$ ) ranges in weighted frequency $f_{G}=5.28 \mathrm{mHz}\left(T_{G}=189 \mathrm{~s}\right), f_{y}=$ $5.65 \mathrm{mHz}\left(T_{y}=177 \mathrm{~s}\right), f_{x}=6.63 \mathrm{mHz}\left(T_{x}=151 \mathrm{~s}\right)$. The mean value $\bar{f}=5.85 \mathrm{mHz}(\bar{T}=171 \mathrm{~s})$. The estimated Doppler shift is then $D f_{y}=0.37 \mathrm{mHz}$ and $D f_{x}=$ $1.35 \mathrm{mHz}$, and the wavelength in the $y$-component on Freja should be shortened by multiplying with the coefficient 0.93 and in the $x$-component with the coefficient 0.80 .

The amplitude relations (Table 1) are $A_{y}=0.18 \mathrm{nT}$, $A_{x}=0.11 \mathrm{nT}$ and $A_{G}=0.07 \mathrm{nT}$. Then $R_{G / F}=0.44$. The total wave in the $(x, y)$-plane on Freja (compound oscillation at both coordinates) is also $L$-polarized. The cross-spectral phase $\Psi_{y x}(\bar{f})=0.14 \mathrm{rad} \approx 0.045 \pi$ (Fig. 2c, Table 1) also shows the phase lag of the signal on the ground. This phase difference value can be uncertain owing to the possible signal distortion at lower frequencies due to polynomial filtration.

c. The first spectral component in the Pc3 range has weighted frequencies $f_{G}=23.44 \mathrm{mHz}\left(T_{G}=42.7 \mathrm{~s}\right)$, $f_{y}=23.23 \mathrm{mHz}\left(T_{y}=43.0 \mathrm{~s}\right), \quad f_{x}=23.46 \mathrm{mHz}\left(T_{x}=\right.$ $42.6 \mathrm{~s}), \bar{f}=23.37 \mathrm{mHz}(\bar{T}=42.8 \mathrm{~s})$. A slight Doppler shift is indicated, $D f_{y}=-0.21 \mathrm{mHz}, D f_{x}=0.02 \mathrm{mHz}$,

Table 1. Estimated amplitude relations between $x$-Freja $\left(A_{x}\right)$, $y$-Freja $\left(A_{y}\right)$, and the ground measurement at Budkov $\left(A_{G}\right)$, the transmission coefficient $R_{G / F}$, and the phase value of the $y$-Freja and $x$-Budkov signal cross-spectrum, calculated for the mean frequency, $\bar{f}$

\begin{tabular}{lcccccc}
\hline $\begin{array}{l}\text { Event } \\
1993\end{array}$ & $\begin{array}{l}\bar{f} \\
(\mathrm{mHz})\end{array}$ & $\begin{array}{l}A_{x} \\
(\mathrm{nT})\end{array}$ & $\begin{array}{l}A_{y} \\
(\mathrm{nT})\end{array}$ & $\begin{array}{l}A_{G} \\
(\mathrm{nT})\end{array}$ & $R_{G / F}$ & $\begin{array}{l}\Psi_{y x} \\
(\mathrm{rad})\end{array}$ \\
\hline 17 September & 5.85 & 0.11 & 0.18 & 0.07 & 0.44 & 0.14 \\
& 13.78 & 0.16 & 0.22 & 0.12 & 0.60 & 0.61 \\
& 23.37 & 0.08 & 0.10 & 0.03 & 0.33 & 0.89 \\
& 28.15 & 0.05 & 0.05 & 0.02 & 0.40 & 0.88 \\
18 September & 10.63 & 0.11 & 0.16 & 0.09 & 0.65 & 0.37 \\
& 19.21 & 0.08 & 0.03 & 0.03 & 0.50 & 0.96 \\
& 23.58 & 0.03 & 0.02 & 0.03 & 0.80 & 0.81 \\
13 May & 20.47 & 0.31 & 0.55 & 0.08 & 0.18 & -0.31 \\
\hline
\end{tabular}

and the coefficients of the wavelength change are 1.01 in the $y$-Freja and 0.99 in the $x$-Freja components. The Doppler effect is negligible.

The amplitude relations are $A_{y}=0.10 \mathrm{nT}, A_{x}=$ $0.08 \mathrm{nT}, A_{G}=0.03 \mathrm{nT}$. Then coefficient $R_{G / F} \approx 0.33$. The total wave of this component in $(x, y)$-Freja is also $L$-polarized. The cross-spectral phase $\Psi_{y x}(\bar{f})=0.89 \mathrm{rad}$ (Fig. 2c, Table 1), the phase lag of the ground signal thus being $0.89 \mathrm{rad} \approx 0.28 \pi$.

d. The second spectral component in the $\mathrm{Pc} 3$ range has weighted frequencies $f_{G}=27.87 \mathrm{mHz}\left(T_{G}=35.9 \mathrm{~s}\right), f_{y}$ $=28.48 \mathrm{mHz}\left(T_{y}=35.1 \mathrm{~s}\right), \quad f_{x}=28.35 \mathrm{mHz} \quad\left(T_{x}=\right.$ $35.3 \mathrm{~s}), \bar{f}=28.15 \mathrm{mHz}(\bar{T}=35.5 \mathrm{~s})$. A slight Doppler shift is also indicated, $D f_{y}=0.61 \mathrm{mHz}, D f_{x}=0.48 \mathrm{mHz}$, and the possible shortening of the wavelengths in both the $y$-and $x$-Freja components would then be indicated by the coefficient $\approx 0.98$.

The amplitudes in both coordinate components on Freja are close, $A_{y} \approx A_{x}=0.05 \mathrm{nT}, A_{G}=0.02 \mathrm{nT}$, and coefficient $R_{G / F} \approx 0.4$ (Table 1 ). The compound total wave in the $(x, y)$-plane on Freja is also $L$-polarized, and the cross-spectral phase $\Psi_{y x}(\bar{f})=0.88 \mathrm{rad} \approx 0.28 \pi$ (Fig. 2c, Table 1). Nearly the same phase lag of the ground signal has been observed in both these spectral components in the $\mathrm{Pc} 3$ range.

e. According to the procedure described in Sect. 3, the estimation of the Doppler frequency shift in the predominant spectral component has been applied to illustrate the characteristics of both wave modes in the $(x, y)$-plane on Freja. They arise owing to the standing oscillation along the magnetic field lines. For stationary frequency $f_{G}=13.83 \mathrm{mHz}$ the coordinate components of wave-vectors $\kappa_{c}$ and $\kappa_{s}, k_{c x}, k_{s x}$ and $k_{c y}=k_{s y}=k_{y}$ were calculated using Eqs. (11-15), and are shown in Table 2.

A very small azimuthal wave number (Eq. 16) was found, $m=-2$, and in agreement with Southwood (1974) and Yumoto et al. (1985) this type of oscillation may be coupled with the compressional waves propagating through the magnetosphere. The sign of the $m$-value determines the azimuthal direction of propagation of the oscillation structure $(m>0$ to the east, and $m<0$ to the west). Angle $\alpha$ (Eq. 17) indicates deflection of the shear Alfvén mode wavefront from the positive direction of the $x$-axis $(\alpha>0$ to the east). Thus for the predominant spectral component with $f=13.83 \mathrm{mHz}$ the shear mode propagated in the northwest direction in the $(x, y)$-plane with $\alpha \approx 53^{\circ}$ and with a velocity

Table 2. Estimated dynamical characteristics of both the wave $c$ and $s$-modes on Freja and the ground-signal weighted frequency $f_{G}$ : coordinate components of the wave vectors, azimuthal wave number $m$ and the deflection angle $\alpha$ of the $s$-wave front (positive to the east)

\begin{tabular}{lllllll}
\hline $\begin{array}{l}\text { Event } \\
1993\end{array}$ & $\begin{array}{l}f_{G} \\
(\mathrm{mHz})\end{array}$ & $\begin{array}{l}\kappa_{y} \\
\left(\mathrm{~km}^{-1}\right)\end{array}$ & $\begin{array}{l}k_{s x} \\
\left(\mathrm{~km}^{-1}\right)\end{array}$ & $\begin{array}{l}k_{c x} \\
\left(\mathrm{~km}^{-1}\right)\end{array}$ & $m$ & $\begin{array}{l}\alpha \\
(\mathrm{deg})\end{array}$ \\
\hline 17 Sept. & 13.83 & $-1.33 \mathrm{E}-4$ & $1.76 \mathrm{E}-4$ & $-8.99 \mathrm{E}-4$ & -2.0 & 53 \\
18 Sept. & 10.50 & $-8.18 \mathrm{E}-5$ & $1.25 \mathrm{E}-4$ & $6.70 \mathrm{E}-4$ & -1.2 & 57 \\
13 May & 20.20 & $-3.29 \mathrm{E}-4$ & $5.96 \mathrm{E}-4$ & $-3.67 \mathrm{E}-4$ & -4.8 & 61 \\
\hline
\end{tabular}


$\approx 395 \mathrm{~km} / \mathrm{s}$, determined by means of $k_{y}, k_{s x}$. The big difference between the wave- and spacecraft- velocities $(|\mu| \approx 7 \mathrm{~km} / \mathrm{s})$ in the both components is a consequence of the nearly negligible Doppler effect estimated.

\subsection{September 1993, 02:32:01-02:38:01 UT}

Other details on $K_{p}=1_{-}, A_{p}=4$; Freja altitude $=1766$ to $1718 \mathrm{~km}$. The Freja footprint was $\phi \approx 58.61^{\circ}$ to $48.45^{\circ}, \lambda \approx 1.20^{\circ}$ to $17.96^{\circ}$.

We shall briefly try to illustrate similar characteristics as analyzed already, for this pulsation event, also occurring in the night hours. The analyzed section of the pulsation record in the $x$ - and $y$-Freja components and on the ground ( $x$-Budkov) is shown in Fig. $4 \mathrm{a}-\mathrm{c}$. The associated display of the $y$-Freja and $x$-Budkov signal power-spectra is in Fig. 5a. Their cross-spectral module and cross-spectral phase in dependence on frequency are shown in Fig. 5b, c. In this case Alfvéntype $L$-polarization of the total $(x, y)$-Freja wave has also been observed in all the principal spectral components of the pulsation. Therefore, only the results of the $y$-Freja and $x$-Budkov spectral analyses will be shown for the three principal spectral components in the Pc4 and Pc3 frequency range.

a. The predominant spectral component ranges in weighted frequency $f_{G}=10.50 \mathrm{mHz}\left(T_{G}=95.2 \mathrm{~s}\right)$,

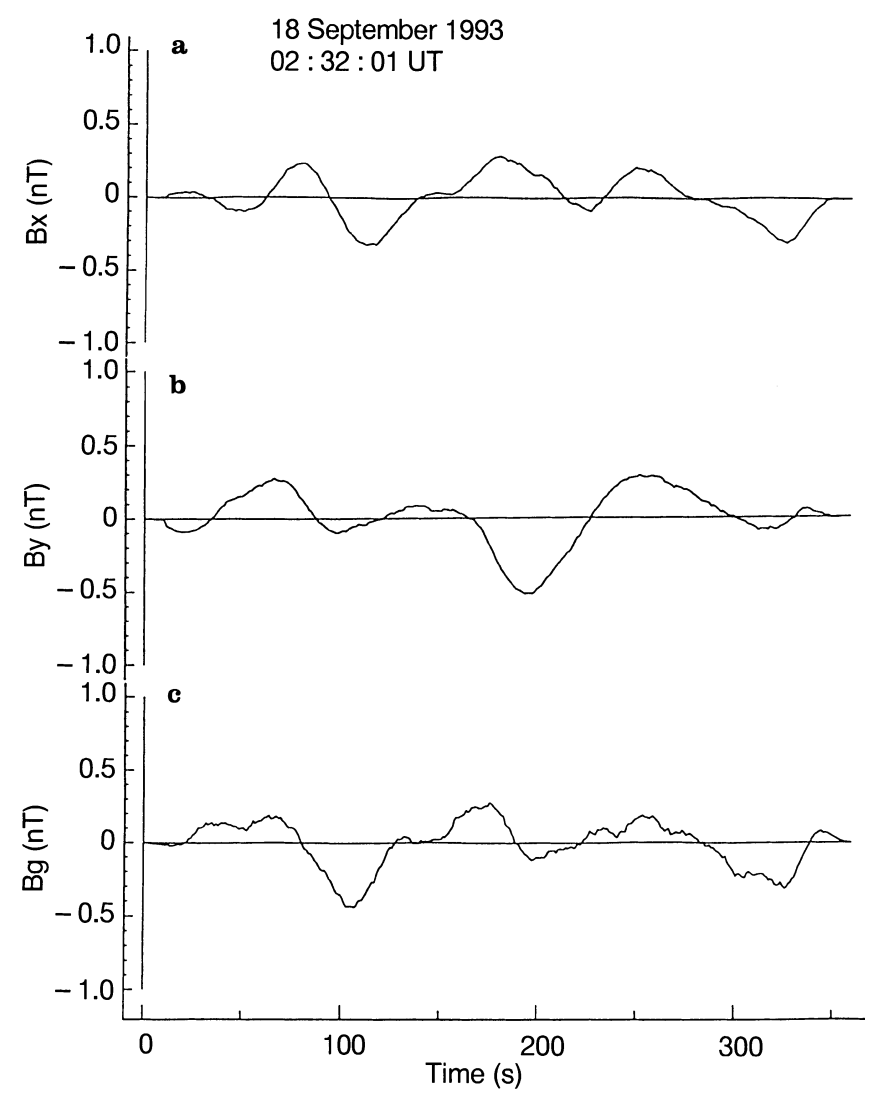

Fig. 4a-c. The simultaneous modified B-field signal records for 18 Sept. 1993, 02:32:01-02:38:01 UT, a $x$-Freja, b $y$-Freja, c $x$-Budkov

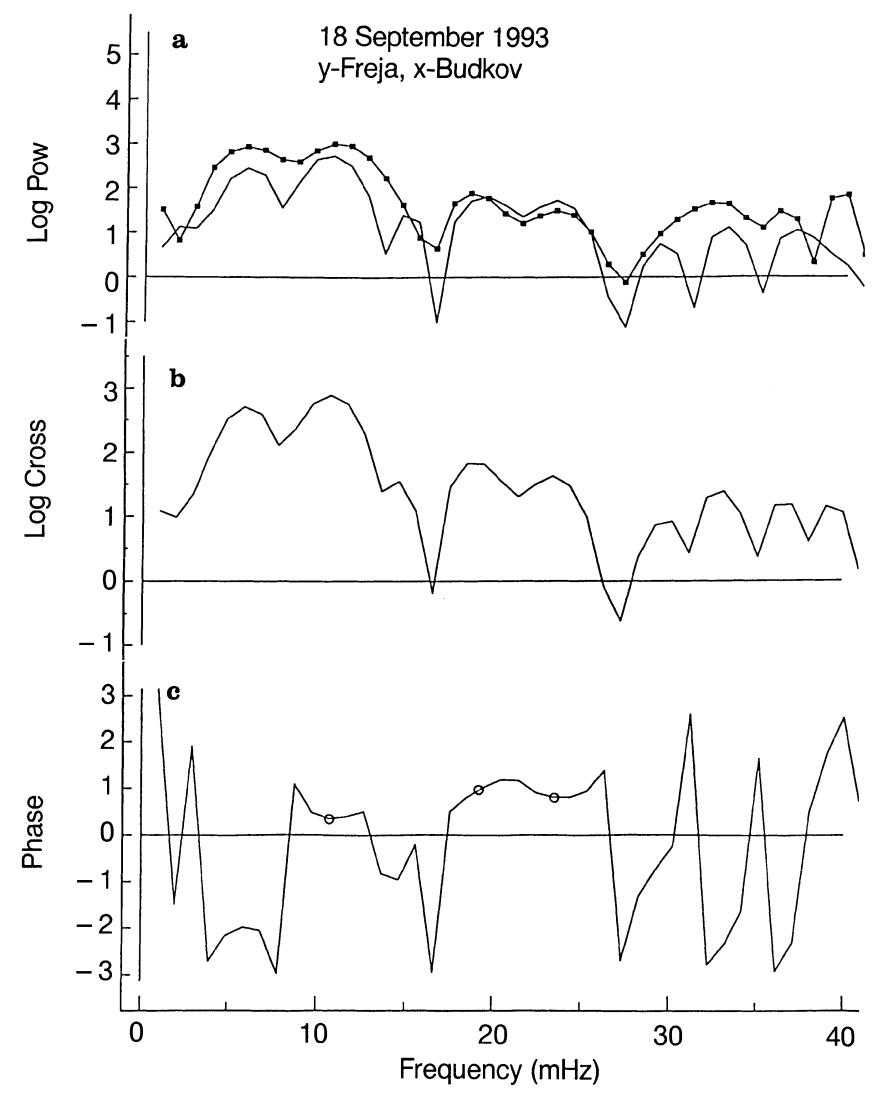

Fig. 5a-c. Frequency dependences for the event of 18 Sept. 1993, analogous to Fig. 2 for $y$-Freja and $x$-Budkov records: a the powerspectra, b the real modulus of the cross-spectrum (both on $\log n \mathrm{~T}^{2} / \mathrm{Hz}$ ), $\mathbf{c}$ the cross-spectral phase (on rad)

$f_{y}=10.66 \mathrm{mHz}\left(T_{y}=93.8 \mathrm{~s}\right), \quad f_{x}=11.05 \mathrm{mHz} \quad\left(T_{x}=\right.$ $90.5 \mathrm{~s})$. The mean value $\bar{f}=10.63 \mathrm{mHz} \quad(\bar{T}=94.1 \mathrm{~s})$. The Doppler shift in the $y$-Freja and $x$-Freja frequencies can then be estimated as $D f_{y} \approx 0.16 \mathrm{mHz}$, $D f_{x} \approx 0.55 \mathrm{mHz}$, indicating a shortening of the wavelengths in both coordinates, with the coefficient 0.98 in $y$-Freja and 0.95 in $x$-Freja.

The amplitude relations are presented in Table 1 together with the cross-spectral phase $\Psi_{y x}(\bar{f})=$ $0.37 \mathrm{rad}$. A phase lag of $0.37 \mathrm{rad} \approx 0.12 \pi$ of the signal on the ground is thus indicated.

b. The other spectral component is considered with weighted frequencies $f_{G}=19.52 \mathrm{mHz}\left(T_{G}=51.2 \mathrm{~s}\right)$, $f_{y}=19.10 \mathrm{mHz}\left(T_{y}=52.4 \mathrm{~s}\right), \quad f_{x}=18.84 \mathrm{mHz}\left(T_{x}=\right.$ $53.1 \mathrm{~s}), \quad \bar{f}=19.21 \mathrm{mHz}(\bar{T}=52.1 \mathrm{~s})$. Then $D f_{y} \approx$ $-0.42 \mathrm{mHz}, D f_{x} \approx-0.68 \mathrm{mHz}$, indicating a lengthening of the wavelengths in both coordinates, with coefficient of 1.02 in $y$-Freja and 1.04 in $x$-Freja.

The amplitude relations are also presented in Table 1. Here $A_{y}<A_{x}$, but the total wave in the $(x, y)$-plane on Freja is $L$-polarized and the cross-spectral phase (Fig. 5c, Table 1) is $\Psi_{y x}(\bar{f})=0.96 \mathrm{rad}$. A phase lag of $0.96 \approx 0.31 \pi$ of the signal on the ground is thus indicated.

c. The spectral component in the Pc3 range is now considered with weighted frequencies $f_{G}=23.41 \mathrm{mHz}$ 
$\left(T_{G}=42.7 \mathrm{~s}\right), f_{y}=23.67 \mathrm{mHz}\left(T_{y}=42.2 \mathrm{~s}\right), f_{x}=23.81$ $\mathrm{mHz} \quad\left(T_{x}=42.0 \mathrm{~s}\right), \bar{f}=23.58 \mathrm{mHz} \quad(\bar{T}=42.4 \mathrm{~s})$. Then $D f_{y} \approx 0.26 \mathrm{mHz}, D f_{x} \approx 0.40 \mathrm{mHz}$, indicating a shortening of the wavelengths in both coordinates with coefficient 0.99 in $y$-Freja and 0.98 in $x$-Freja.

The amplitude relations in Table 1 also show that $A_{y}<A_{x}$, the value $A_{G}$ being relatively high. Considering $A_{G}$ in the $x$-Budkov component, predominant on the ground, it will be better to calculate the amplitude ratio $R_{G / F}$ using the formula

$$
R_{G / F} \approx A_{G} / \sqrt{\left(A_{x}^{2}+A_{y}^{2}\right)}
$$

hence $R_{G / F}=0.8$. However, the compound total wave in the $(x, y)$-plane on Freja is also $L$-polarized, and the cross-spectral phase (Fig. 5c, Table 1) $\Psi_{y x}(\bar{f})=0.81 \mathrm{rad}$ also indicates a phase lag of $0.81 \mathrm{rad} \approx 0.26 \pi$ of the signal on the ground.

d. Using the procedure of Sect. 3, the dynamic characteristics of both wave modes of the predominant spectral component in the $(x, y)$-plane on Freja were calculated for stationary frequency $f_{G}=10.50 \mathrm{mHz}$, and are given in Table 2. Also a very small azimuthal wave number, $m=-1.2$, was found, and the shear Alfvén mode also propagated in the northwest direction with $\alpha=57^{\circ}$ and velocity $\approx 440 \mathrm{~km} / \mathrm{s}$. The results for both pulsation events are very similar.

\subsection{May 1993, 09:20:00-09:26:00 UT}

Other details include $K_{p}=0_{+}, \quad A_{p}=10 ; \quad$ Freja altitude $=1742$ to $1659 \mathrm{~km}$. The Freja footprint was $\phi \approx 53.66^{\circ}$ to $48.32^{\circ}, \lambda \approx-3.34^{\circ}$ to $4.13^{\circ}$.

This daytime pulsation event was also analyzed with respect to the amplitude and resonance characteristics in Prikner (1996). The conjugacy in longitude of the measurement is poor $\left(\Delta \lambda>10^{\circ}\right)$. It is presented here to illustrate possible distortions of the spectral and/or crosspectral characteristics by this $\Delta \lambda$-shift.

The signal records in the $x$-Freja, $y$-Freja and $x$-Budkov components are displayed in Fig. $6 \mathrm{a}-\mathrm{c}$. The associated power-spectra in the $y$-Freja and $x$-Budkov components are shown in Fig. 7a. The real cross-spectral module and phase in dependence on frequency are in Fig. 7b, c. The power-spectra in Fig. 7a show that the predominant spectral components on Freja and on the ground are different $\left(f_{G}=20.20 \mathrm{mHz}\right.$ on the ground, $f_{y}=7.17 \mathrm{mHz}$ on Freja).

Let us restrict ourselves to the analysis of the ground predominant spectral component with weighted frequencies $f_{G}=20.20 \mathrm{mHz}\left(T_{G}=49.5 \mathrm{~s}\right), f_{y}=20.91 \mathrm{mHz}$ $\left(T_{y}=47.8 \mathrm{mHz}\right), \quad f_{x}=20.47 \mathrm{mHz}\left(T_{x}=49.4 \mathrm{~s}\right), \quad \bar{f}=$ $20.47 \mathrm{mHz}(\bar{T}=48.9 \mathrm{~s})$. The Doppler shifts, $D f_{y} \approx$ $0.71 \mathrm{mHz}, D f_{x} \approx 0.02 \mathrm{mHz}$, indicate shortening of wavelengths in both components with coefficients 0.97 in $y$ Freja and 0.99 in $x$-Freja.

The amplitude relations in Table 1 indicate wave damping in the ionosphere with coefficient (Eq. 18),

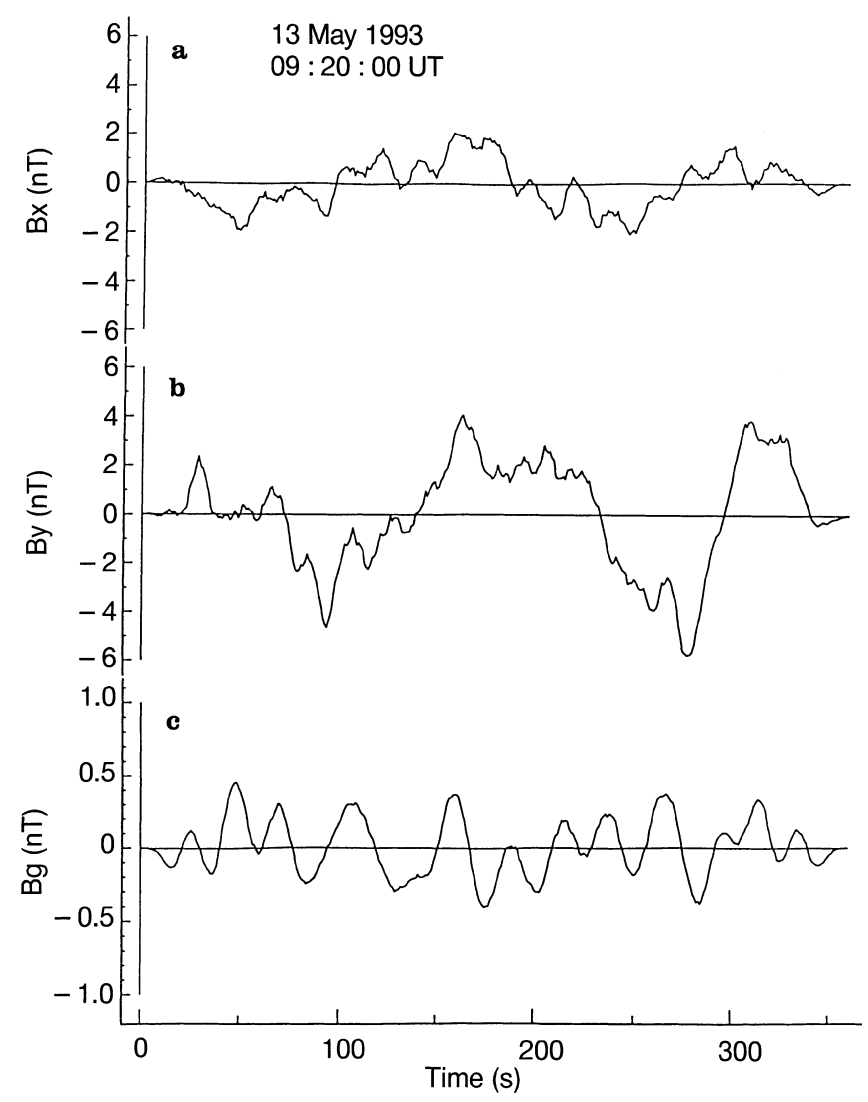

Fig. 6a-c. The simultaneous modified B-field signal records for 13 May 1993, 09:20:00-09:26:00 UT, a $x$-Freja, b $y$-Freja, c $x$-Budkov

$R_{G / F}=0.18$. Also this small value is evidence that, on the ground, only the residual part of the Freja signal was recorded (different mixed signals at both points of measurement), and/or the ionospheric damping was much greater during this daytime event.

The compound total wave in the $(x, y)$-plane on Freja was also $L$-polarized and the cross-spectral phase $\Psi_{y x}(\bar{f})=-0.31 \mathrm{rad} \approx-0.1 \pi$. This indicates a signal phase lag in the $y$-Freja, and could also be the consequence of the longitudinal shift from conjugacy.

The dynamic characteristics of both wave modes in the $(x, y)$-plane on Freja calculated for this grounddominant spectral component are also in Table 2. The shear Alfvén mode with small $m \approx-4.8$ (uncertain due to a poor signal coherence) also propagated in the northwest direction with $\alpha \approx 61^{\circ}$ and a velocity of around $186 \mathrm{~km} / \mathrm{s}$. Care needs to be exercised in interpreting the estimated wave number and velocity for the 13 May event, since it is not clear that the same wave was observed at both sites.

\section{Discussion and conclusion}

Two nearly conjugate and one quasi-conjugate simultaneous Freja-Budkov intervals of ULF pulsation measurements were studied. All three events occurred during quiet geomagnetic field conditions $\left(K_{p}<1\right)$. Due to the 


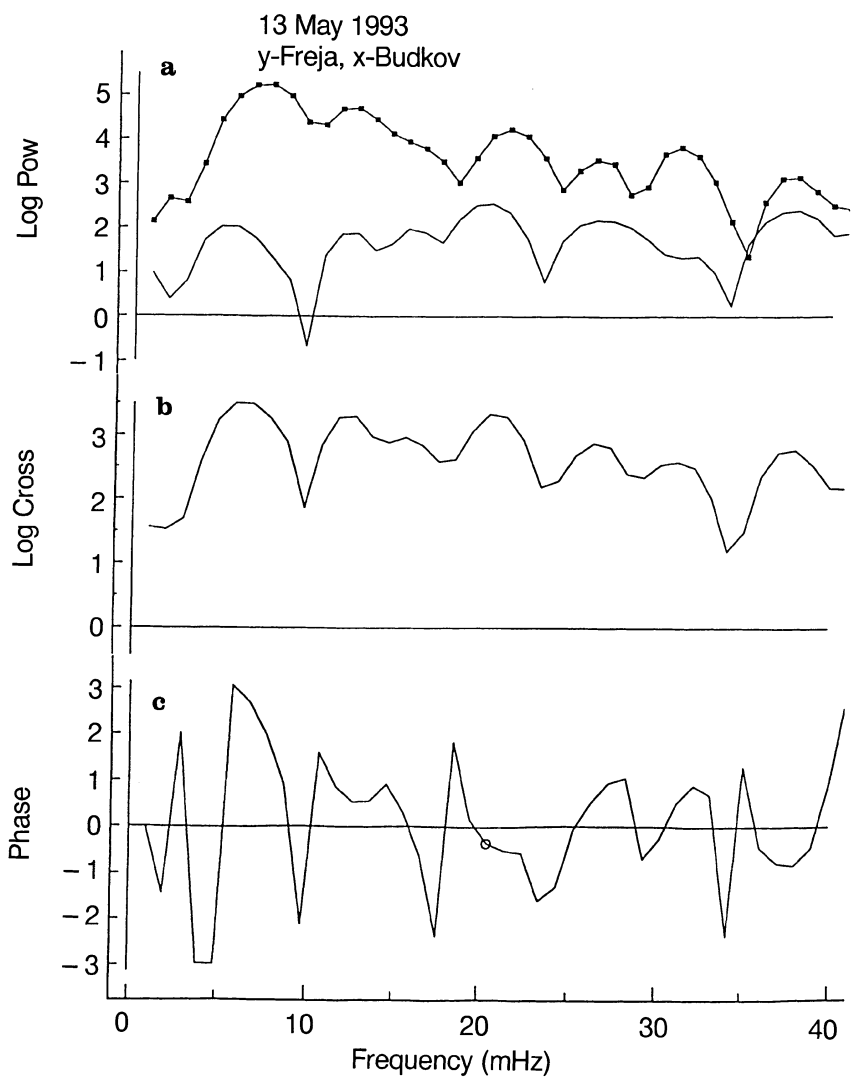

Fig. 7a-c. Frequency dependences for the event of 13 May 1993 , analogous to Fig. 2 for $y$-Freja and $x$-Budkov records: a the powerspectra, b the real modulus of the cross-spectrum (both on $\log n \mathrm{~T}^{2} / \mathrm{Hz}$ ), $\mathbf{c}$ the cross-spectral phase (on rad)

rare coincidence of pulsation events at both points of measurement during the very short intervals of conjugacy, the number of events studied was small.

In accordance with Prikner (1996) the results show that a wide longitudinal region of the $\mathrm{Pc} 3-4$ type oscillation structure may exist at latitude $\phi \approx 50^{\circ}$. According to Singer et al. (1982), poloidal Pc4 oscillations are usually measured in a narrow belt in the radial direction, $\Delta R \approx 1 R_{E}$, while Engebretson et al. (1992) found that the longitudinal extent of poloidal Pc4 is nearly $8 \mathrm{~h}$ in LT. According to Yumoto and Saito (1983) and Yumoto et al. (1985), low-latitude Pc3-4 may have limited longitudinal extent, $\Delta \lambda \approx \pm 10^{\circ}$. Our measurements on Freja in longitudinal extent $\Delta \lambda \approx 17^{\circ}$ (the night-time events of 17 and 18 September 1993) did not show a substantial deterioration of the FFT spectra in comparison with the ground, but a "collection" of different signals or signals with changing frequency could be expected in the signal spectrum for pulsations measured during the Freja's 6-min section of orbit. A more precise comparison of the ground-satellite correspondence (or coherence) could be obtained with a peak-to-peak comparison of the Freja and Budkov time series.

Also the latitude span during the 6-min Freja measurements in the 17 and 18 September events was very wide, $\Delta \phi \approx 10^{\circ}$ around $\phi \approx 50^{\circ}\left(L_{B} \approx 2.3\right.$ for
Budkov), which indicates a wide latitude extent of the oscillation region during these night events. For the daytime Pc3 pulsations, e.g., according to Walker et al. (1979) and Ziesolleck et al. (1993), the typical extent of the resonance region is only $0.2<\Delta L<0.8\left(\Delta \phi \approx 1^{\circ}\right.$ in the north-south direction at $\phi \approx 70^{\circ}$ ), which represents $250 \mathrm{~km}-1500 \mathrm{~km}$ or more in the ionosphere.

The spectral and cross-spectral analysis provided an indication of the signal frequency content. The amplitude and phase characteristics of the limiting low-frequency components (Pc5 range) could not be analyzed with sufficient accuracy due to inevitable application of the signal filtration technique. The spectra of the components with small power are also expected to be distorted. Therefore, only the predominant and several principal spectral components from the mid Pc3-4 zone were selected and analyzed.

However, both the signals on 17 and 18 September events were highly coherent in selected frequencies. According to Poulter and Allan (1985) the latitudinal amplitude and period variations for signals of the transient-type ULF pulsations behave in a similar manner to the amplitude and phase variations for monochromatic resonances determined by Hughes and Southwood (1976b).

The general effect of the atmosphere and ionosphere are a smoothing and spreading of the amplitude and phase latitudinal variation seen on the ground coupled with a rotation of magnetospheric polarization through $90^{\circ}$. Also the latitudinal variation of periods is smeared on ground magnetometers due to the effects of spatial integration. The ground oscillation is further spread in latitude (Poulter and Allan, 1985). The latitudinal period variation deduced on the ground is a good reproduction of the ionospheric variation over the central portion of the current region, and becomes constant outside. Considering predominant spectral component of the highly coherent signal both on Freja and at Budkov, we can assume the Freja signal was measured inside the central portion of the resonance region and the frequency should correspond to this on the ground. Then a mutual difference between frequencies in the $x$ - and $y$-components measured at the one point on Freja and their differences from the groundmeasured frequency should indicate the Doppler effect in the $(x, y)$-plane with a sufficient accuracy.

The detailed frequency analysis of selected spectra showed that the Doppler frequency shifts in the $(x, y)$ plane on Freja were small but not negligible. The estimated values are of the order of $1 \mathrm{mHz}$ (mostly of fractions of $\mathrm{mHz}$ ), indicating a change of wavelength with coefficients $0.93-1.02$ in $y$-Freja and $0.80-1.04$ in $x$-Freja. The values estimated here are perceptibly lower than the estimates obtained for the daytime events analyzed in Prikner (1996).

The amplitude relations and phase shifts of the signal on the ground regarding the signal on Freja are shown in Table 1. All the studied spectral components of the pulsation events were Alfvén-type L-polarized in the $(x, y)$-plane. Considering the $90^{\circ}$ counterclockwise rotation of the polarization axis (Nishida, 1964; Hughes 
1974), the $y$-Freja and $x$-Budkov signal cross-spectra can provide important information on the phase shift of the signal field during propagation through the ionosphere to the ground (or rather the phase shift in the standing oscillation between the upper ionosphere and the ground).

To obtain a reliable phase estimation for a particular pulsation spectral component on Freja and on the ground, it is necessary to ensure precise synchronization of measurements, on the one hand, and reliable phase calibration of the signal records in both positions of measurement, on the other. For example, a constant time shift of one recorder causes a rapid increase of the cross-spectral phase value with increasing frequency. The accuracy of the record initial time on the ground was within 1 second (assuming precise time record on Freja).

In the principal spectral components of the samples with good conjugacy (September 17, 18) the phase lag of the signal on the ground relative to the signal on Freja has been estimated. In the particular pulsation component of the 18 September event with $f=19.21 \mathrm{mHz}$ (Table 1) this represents a maximum time shift of $<8 \mathrm{~s}$. It is claimed that the phase lag of the signal on the ground can be of natural origin, even if a slight inaccuracy in phase calibrations of the both signal records is admitted. The author did not find similar signal phase shifts studied hitherto on spacecraft in high-altitude and stationary orbits in the references.

The model computations in Hughes and Southwood (1976b) give an idea about orientation of tilt of the horizontal polarization ellipse near the resonance region and its changes between the ionosphere and the ground, assuming straight and uniform ambient magnetic field in the $z$-direction and with the cold plasma density varying in the $x$-direction. The rotation of the polarization axis need not to be exactly $90^{\circ}$. The modelled deviation depends on the latitude profile around the resonance region and it ranged roughly inside the $\pm 15^{\circ}$ limits $( \pm 0.26 \mathrm{rad})$. The $x$-Budkov phase lags with respect to $y$-Freja $\left(\Psi_{y x}\right.$ in Table 1$)$ range between $8^{\circ}$ to $55^{\circ}$. It should indicate counterclockwise rotation less than $90^{\circ}$ in prevailing spectral components of the 17 and 18 September events. Such deviations can be a consequence of the arbitrary non-uniform horizontal conductivity distribution and also the wave propagation in noncoplanar magnetic field geometries (Glassmeier and Junginger, 1987; Glassmeier, 1988).

The amplitude relations between the ground and satellite (Table 1) in the samples with good conjugacy are in good agreement with, e.g., Glassmeier (1988) and Wedeken et al. (1984), when the transmission coefficient ranged roughly within the limits 0.2 to 0.9 . Here the coefficient $R_{G / F}$ has been estimated between $0.3-0.8$. This is also in good agreement with the results of the numerical simulation of ionospheric filtration (e.g., Prikner and Vagner, 1983; Prikner, 1996).

Deviations in amplitude relations and the anomalous phase shift at the ground in the predominant spectral component of the 13 May event can be attributed to poor conjugacy in geographical longitude. Nevertheless, phase lags of the ground signal can also be found in close frequency spectral components (see Fig. 7a-c). We can assume that different signals were mixed in both points of measurement. The simulation technique (e.g., Prikner and Vagner, 1983) using different models of the ionosphere can be applied to simultaneous pulsation samples with good conjugacy.

Small azimuthal wave numbers for predominant pulsation spectral components are apparent in the dynamical characteristics of the shear and compressional wave modes in the $(x, y)$-plane on Freja. The shear wave mode of the standing wave oscillation structure in the $(x, y)$-plane propagated in the north-west direction. The estimates of the Doppler wavelength change agree with the direction of propagation of the resultant wave, compound of both the wave modes, in the $(x, y)$-plane. The coordinate components of $\mathrm{K}_{c}$ and $\mathrm{K}_{s}$ for predominant components in the three analysed events are in Table 2. This type of oscillation, taking into account the models of global resonances (Allan et al., 1987; Allan and Poulter, 1989), can be excited by broadband compressional power.

Acknowledgements. The author wishes to thank H. Lühr and M. Rother of the Institut für Geophysik und Meterologie, Technische Universität, Braunschweig, Germany, for providing unique records of the Freja ULF measurements and helpful comments regarding data processing. The author wishes to thank $\mathrm{M}$. Konečný of the Geophysical Institute, Acad. Sci. Czech Republic, Prague, for providing the Budkov pulsation data.

The Editor in chief thanks two referees for their help in evaluating this paper.

\section{References}

Allan, W., and E. M. Poulter, Damping of magnetospheric cavity modes: a discussion, J. Geophys. Res., 94, 11843-11853, 1989.

Allan, W., E. M. Poulter, and S. P. White, Hydromagnetic wave coupling in the magnetosphere - magnetic fields and Poynting fluxes, Planet. Space Sci., 35, 1181-1192, 1987.

Anderson, B. J., Statistical studies of Pc3-5 pulsations and their relevance for possible source mechanisms of ULF waves, Ann. Geophysicae, 11, 128-143, 1993.

Baumjohann, W., N. Sckopke, J. LaBelle, B. Klecker, H. Lühr, and K.-H. Glassmeier, Plasma and field observations of a compressional Pc5 wave event, J. Geophys. Res., 92A, 12203-12212, 1987.

Cummings, W. D., R. J. O'Sullivan, and P. J. Coleman, Jr., Standing Alfvén waves in the magnetosphere, J. Geophys. Res., 74, 778-793, 1969.

Engebretson, M. J., D. L. Murr, K. N. Erickson, R. J. Strangeway, D. M. Klumpar, S. A. Fuselier, L. J. Zanetti, and T. A. Potemra, The spatial extent of radial magnetic pulsation events observed in the dayside near synchronous orbit, J. Geophys. Res., 97, 13741-13758, 1992.

Engebretson, M. J., L. J. Zanetti, T. A. Potemra, and M. H. Acuna, Harmonically structured ULF pulsations observed by the AMPTE CCE magnetic field experiment, Geophys. Res. Lett., 13, 905-908, 1986.

Freja magnetic field experiment team, Magnetic field experiment on the Freja satellite, Space Sci. Rev., 70, 465-482, 1994.

Glassmeier, K.-H., Reconstruction of the ionospheric influence on ground-based observations of a short-duration ULF pulsation event, Planet. Space Sci., 36, 801-817, 1988.

Glassmeier, K.-H., and H. Junginger, Concerning the ionospheric modification of magnetospheric hydromagnetic waves: case studies, J. Geophys. Res., 92, 12213-12219, 1987. 
Hughes, W. J., The effect of the atmosphere and ionosphere on long period magnetospheric micropulsations, Planet. Space Sci., 22, 1157-1172, 1974.

Hughes, W. J., and D. J. Southwood, The screening of micropulsation signals by the atmosphere and ionosphere, J. Geophys. Res., 81, 3234-3240, 1976a.

Hughes, W. J., and D. J. Southwood, An illustration of modification of geomagnetic pulsation structure by the ionosphere, J. Geophys. Res., 81, 3241-3247, 1976 b.

Nishida, A., Ionospheric screening effect and storm sudden commencement, J. Geophys. Res., 69, 1861-1874, 1964.

Poulter, E. M., and W. Allan, Transient ULF pulsation decay rates observed by ground based magnetometers: the contribution of spatial integration, Planet. Space Sci., 33, 607-616, 1985.

Prikner, K., Characteristics of the mid-latitude ionosphere suitable for modelling ionospheric filtration of ULF waves, Stud. Geoph. Geod., 28, 306-315, 1984.

Prikner, K., Spatial features of mid-latitude field line resonances from simultaneous ground-satellite measurements, Ann. Geophysicae, 14, 699-706, 1996.

Prikner, K., and V. Vagner, Numerical modelling of the ionospheric filtration of an ULF micropulsation signal, Stud. Geoph. Geod., 27, 173-190, 1983.

Prikner, K., and V. Vagner, Vertical ULF (Pc1) changes of modal wave characteristics in the midlatitude ionosphere (Part 1-the complex index of refraction), Trav. Géophys. XXXIII, Trav. Inst. Géophys. Acad. Tchécosl. Sci., 610, pp. 327-352, 1985a.

Prikner, K., and V. Vagner, Vertical ULF (Pc1) changes of modal wave characteristics in the midlatitude ionosphere (Part 2Polarization and energy characteristics), Trav. Géophys., XXXIII, Travaux Inst. Géophys. Acad. Tchécosl. Sci., 611, pp. 353-370, 1985b.

Prikner, K., and V. Vagner, Changes in the transmissibility of the mid-latitude ionosphere related to the ULF (Pc1) signal, Trav. Géophys., XXXIV, Travaux Inst. Géophys. Acad. Tchécosl. Sci., 618, pp. 135-161, 1986.
Samson, J. C., C. L. Waters, F. W. Menk, and B. J. Fraser, Fine structure in the spectra of low-latitude field line resonances, Geophys. Res. Lett., 22, 2111-2114, 1995.

Singer, H. J., W. J. Hughes, and C. T. Russell, Standing hydromagnetic waves observed by ISEE1 and 2: radial extent and harmonic, J. Geophys. Res., 87A, 3519-3529, 1982.

Southwood, D. J., Some features of field line resonance in the magnetosphere, Planet. Space Sci., 22, 483-491, 1974.

Takahashi, K., and R. L. McPherron, Standing hydromagnetic waves in the magnetosphere, Planet. Space Sci., 32, 1343-1359, 1984.

Walker, A. D. M., R. A. Greenwald, A. Korth, and G. Kremser, STARE and GEOS-2 observations of a storm time Pc5 ULF pulsations, J. Geophys. Res., 87A, 9135-9146, 1982.

Walker, A. D. M., R. A. Greenwald, W. F. Stuart, and C. A. Green, STARE auroral radar observations of Pc5 geomagnetic pulsations, J. Geophys. Res., 84, 3373-3388, 1979.

Wedeken, U., B. Inhester, A. Korth, K. -H. Glassmeier, R. Gendrin, L. J. Lanzerotti, H. Gough, C. A. Green, E. Amata, A. Pedersen, and G. Rostoker, Ground-satellite coordinated study of the April 5, 1979 events: flux variations of energetic particles and associated magnetic pulsations, J. Geophys., 55, 120-133, 1984.

Yumoto, K., Generation and propagation mechanisms of lowlatitude magnetic pulsations-a review, J. Geophys., 60, 79-105, 1986.

Yumoto, K., and T. Saito, Relation of compressional HM waves at GOES 2 to low-latitude Pc3 magnetic pulsations, J. Geophys. Res., 88, 10041-10052, 1983.

Yumoto, K., T. Saito, S. -I. Akasofu, B. T. Tsurutani, and E. J. Smith, Propagation mechanism of daytime Pc3-4 pulsations observed at synchronous orbit and multiple ground-based stations, J. Geophys. Res., 90, 6439-6450, 1985.

Ziesolleck, C. W. S., B. J. Fraser, F. W. Menk, and P. W. McNabb, Spatial characteristics of low-latitude Pc3-4 geomagnetic pulsations, J. Geophys. Res., 98, 197-207, 1993. 